\title{
EL PLAN DE PARTO COMO DOCUMENTO DE INSTRUCCIONES PREVIAS
}

\section{Marta Busquets Gallego ${ }^{1}$}

Correo electrónico: 1a.busquets@ gmail.com

1. Doctoranda en Estudios de Género en la Universitat Rovira i Virgili

Recibido: 07/03/2019 Aceptado: 12/06/2019

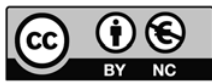




\section{RESUMEN}

\section{Introducción}

En las últimas décadas el plan de parto viene cobrando relevancia como instrumento de manifestación de la voluntad de las mujeres. Ha sido vinculado con resultados obstétricos satisfactorios, pero también como potencial fuente de enfrentamientos entre profesionales y usuarias, así como de decepción en estas últimas en caso de que no pueda ser cumplido.

\section{Objetivo}

El presente artículo pretende esclarecer el estatus legal del plan de parto en España.

\section{Metodología}

Para ello se lleva a cabo un experimento: se redacta un plan de parto extenso, que es elevado a público en sede notarial y finalmente inscrito en el Registro de Voluntades Anticipadas de Cataluña.

\section{Resultados y conclusiones}

De acuerdo con el experimento, el plan de parto es un documento de instrucciones previas, lo cual tiene consecuencias jurídicas en cuanto a su obligatoriedad y aplicación.

Palabras clave: plan de parto; instrucciones previas; voluntades anticipadas; autonomía; consentimiento informado; género. 


\section{ABSTRACT}

\section{Introduction}

In the last decades birth plans have been gaining relevance as instruments of manifestation of the will of women. They have been linked to satisfactory obstetric results but have been also referred to as a source of conflict between professionals and users, as well as a frustration source for the latter in case they cannot be accomplished.

\section{Objectives}

This article aims to clarify the legal status of birth plans in Spain.

\section{Methodology}

An experiment is carried out: an extensive birth plan is written, then notarized and finally registered in the Catalan Register of Advance Directives.

\section{Results and Conclusions}

According to the experiment, birth plans are an advance directive, which has legal consequences regarding their enforceability and application.

Keywords: Birth Plan; Advance Directive; Autonomy; Informed Consent; Gender. 


\section{INTRODUCCIÓN}

Sheila Kitzinger (1983) acuñó el concepto plan de parto como estrategia para contrarrestar la excesiva medicalización del mismo, siendo este un documento escrito donde la embarazada plasma su voluntad tras haber recibido información sobre el embarazo y el parto, de acuerdo con sus valores, deseos personales, expectativas y necesidades particulares (Hasenmiller, 2001).

En el Reino Unido se empezaron a usar los planes de parto en 1993. En el año 2007 el Protocolo para la asistencia al parto normal catalán lo mencionó e incluyó un modelo, también nombrándose en la Estrategia de atención al parto normal española en 2007 y publicándose por primera vez un modelo en la misma en el año 2012 (Suárez-Cortés et al., 2015; Biescas et al., 2017).

De acuerdo con María Suarez-Cortés et al. (2015) los planes de parto influyen positivamente en el proceso, relacionándose su uso con mejores tasas de partos eutócicos, piel con piel o pinzamiento tardío del cordón. Prácticas que además directa e indirectamente disminuyen el gasto sanitario por hospitalizaciones. Además, incrementan la satisfacción, empoderamiento y seguridad de las mujeres. Por todo ello se ha establecido la necesidad de impulsar políticas sanitarias que incentiven su uso.

Los planes de parto fomentan una mejor comunicación y relaciones más respetuosas entre mujeres y profesionales, incrementando a su vez la sensación de control de las primeras permitiendo que se familiaricen con el proceso de parto y sus opciones.

Herninia Biescas et al. (2017) nos alertan de que también surgen tensiones y discrepancias. Sugieren que pueden promover actitudes inflexibles en las mujeres y generar decepción si no se cumple el plan de parto. Los profesionales pueden molestarse y sentir que se cuestiona su experiencia y conocimientos.

Asimismo, las autoras han puesto de manifiesto que mientras la intención original de los planes de parto es permitir que las mujeres elijan y se personalice la atención, abandonando modelos altamente medicalizados. En la actualidad, la institucionalización de esta figura puede contribuir a perpetuar las dinámicas hospitalarias, por ejemplo, porque solo se permiten marcar opciones ya preestablecidas por el centro, a la par que genera una falsa sensación de participación en la mujer. 
A efectos prácticos, las mujeres que desean presentar o elaborar un plan de parto se encuentran con distintos escenarios, que pueden agruparse en tres: se les acepta, no se les acepta o se les acepta únicamente el modelo que ofrece el propio centro donde van a dar a luz. Aun siendo aceptados, a menudo se considera el plan de parto como una simple declaración de intenciones sin mayor trascendencia legal o clínica. El presente artículo pretende esclarecer el estatus legal en España de los planes de parto.

\section{METODOLOGÍA}

En primer lugar, se ha establecido el marco jurídico-legal general aplicable a los derechos de autonomía y consentimiento informado en el embarazo y el parto. A continuación, se ha delimitado el marco legal específico aplicable a los documentos de instrucciones previas, también conocidos como voluntades anticipadas.

Para ello se ha recurrido a metodología jurídica deductiva, partiendo de lo general a lo particular y siguiendo el principio de jerarquía, consultando en primer lugar la legislación vigente, posteriormente la jurisprudencia aplicable y por último la doctrina relacionada (López Escacena, 2011). También se ha recurrido a metodología jurídicofilosófica al incorporarse reflexiones que van más allá del derecho e incluyen moral y ética (Sánchez Zorrilla, 2011).

En particular, con el fin de confirmar el estatus jurídico de instrucciones previas del plan de parto, se llevó a cabo el siguiente experimento jurídico siguiendo los pasos que se relatan a continuación:

Se optó por un plan de parto de redactado propio, en contraposición a las propuestas estandarizadas hospitalarias, consignando en él un amplio abanico de situaciones y posibilidades, ${ }^{1}$ con la intención de que fuera únicamente expresión de la autonomía y la voluntad propias, sin condicionantes externos, sanitarios o formalistas.

Dicho plan de parto se presentó ante notario manifestando la voluntad de elevarlo a público como instrucciones previas, acto que tuvo lugar el día tres de mayo del año dos mil dieciocho.

\footnotetext{
${ }^{1}$ El plan de parto incluía un parto vaginal normal, pero también situaciones consideradas de riesgo como ser estreptococo b positiva, así como parto quirúrgico por cesárea. También se consignaron indicaciones sobre cuidados al bebé.
} 
A continuación, la escritura fue remitida al Registro de Instrucciones Previas de Cataluña para su inscripción. El Registro podía o bien aceptar, confirmando el estatus de instrucciones previas del plan de parto, o rechazar, en cuyo caso debería motivar la incompatibilidad con dicha figura legal.

El documento fue en este caso aceptado e inscrito. Por lo tanto, ha existido un triple control de legalidad: por parte de la autora, posteriormente notarial y finalmente en sede registral.

\section{DESARROLLO ARGUMENTATIVO}

Con el cambio de un modelo basado en el imperialismo médico hacia uno basado en la autonomía de la persona, ${ }^{2}$ los derechos de autonomía y consentimiento informado respecto de las intervenciones médicas se han calificado desde la doctrina y los Tribunales derechos humanos fundamentales de tercera generación, ${ }^{3}$ que buscan proteger la libertad de conciencia y el respeto a la dignidad humana (Reynal Reillo, 2017; Llamas Pombo, 2007).

Desde la consagración del modelo autonomista, la tendencia jurídica ha sido la de asegurar y posibilitar el ejercicio de estos derechos, haciendo siempre una interpretación restrictiva de sus limitaciones (Domínguez Luelmo, 2007).

La ley 41/2002, de 14 de noviembre, básica reguladora de la autonomía del paciente y de derechos y obligaciones en materia de información y documentación clínica establece que toda actuación en el ámbito sanitario requiere del consentimiento libre y voluntario previa información y valoración de opciones de la persona afectada. ${ }^{45}$

\footnotetext{
${ }^{2}$ En el modelo imperialista médico o paternalista, las decisiones las tomaba la persona profesional sanitaria al margen de la voluntad del paciente. En el modelo autonomista se reconoce a la persona como ser individual, racional y libre (Feito, 2016; Reynal Reillo, 2017).

${ }^{3}$ Por ejemplo, la STC 37/2011, de 28 de marzo y en particular la STS 447/2001 de 11 de mayo se refiere a los derechos de autonomía y consentimiento informado como «una de las últimas aportaciones a la teoría de los derechos humanos, consecuencia necesaria y explicación de los clásicos derechos a la vida, a la integridad física y a la libertad de conciencia».

${ }^{4}$ Artículos 8 y 9.

${ }^{5}$ También aplican respecto de los derechos de autonomía y el consentimiento informado el Convenio Europeo de Derechos Humanos y Biomedicina de 1997 (conocido como Convenio de Oviedo), artículos 5 a 9, la Carta de Derechos Fundamentales de la Unión Europea (2000/C 364/01), artículo 3, la Carta Europea de Derechos del Paciente de 2002 (artículos 3 a 5) y también se invoca la Constitución Española de 1978 (artículos 15 a 18).
} 
En términos generales, hay tres excepciones a lo anterior (Hernández Ramos, 2012): riesgos para la salud pública por razones sanitarias, teniendo que ver mayoritariamente con enfermedades contagiosas; riesgo inmediato grave siendo imposible recabar la autorización de la persona o consultar con sus allegados; e incapacidad para consentir.

Advierte Domínguez Luelmo (2007) que las intervenciones sin consentimiento siempre deberán interpretarse de forma restrictiva, extremo que han confirmado los tribunales. ${ }^{6}$ El derecho al rechazo es el corolario sin el cual los derechos de autonomía y consentimiento informado se vaciarían de contenido (Lizarraga Bonelli, 2004).

En concreto, el embarazo y el parto no son momentos limitativos de estos derechos sino de especial protección de acuerdo con el Convenio para la Eliminación de la Discriminación y la Violencia contra la Mujer (CEDAW) y su Recomendación General 24 «Mujer y Salud».

También el comisionado para los derechos humanos del Consejo de Europa se ha expresado en lo relativo a los derechos de las mujeres en el embarazo y el parto. Según manifiesta esta institución, los mecanismos de protección de los derechos humanos han establecido que debe ser garantizado el derecho de las mujeres al consentimiento informado y a tomar decisiones en todas las etapas y momentos del embarazo y parto, enfatizando que ninguna intervención ni procedimiento médicos podrán ser llevados a cabo sin el pleno e informado consentimiento de la mujer (Consejo de Europa, 2017, p. 61).

Las instrucciones previas, también conocidas como voluntades anticipadas, se hallan reguladas en España con carácter básico por la Ley 41/2002, en su artículo 11. Las Comunidades Autónomas disponen de regulaciones propias respetando la base anterior. ${ }^{7}$

A pesar de que tradicionalmente se ha vinculado esta figura exclusivamente a procesos de muerte, el propio artículo de la ley habilita que este instrumento legal se utilice en otras situaciones, estableciendo que es un documento donde una persona mayor de edad, capaz y libre, manifiesta su voluntad de forma anticipada respecto de una situación donde prevé que no será capaz de expresarla personalmente. Incluso permite la

\footnotetext{
${ }^{6 \mathrm{~S}}$ TC 29/2000 FJ 9º STC 157/2002 FJ 8º, STC 20/1990 FJ 4º, STC 57/1994, FJ 7º.

${ }^{7}$ Deberá tenerse en cuenta cuáles son los requisitos de validez en cada Comunidad Autónoma para que un documento sea considerado instrucciones previas o voluntades anticipadas.
} 
designación de representante para tareas de interlocución frente a profesionales de la salud con el objetivo de asegurar el cumplimiento de las instrucciones.

Legalmente se establecen tres límites a lo designado en las instrucciones previas: que contradigan al ordenamiento jurídico, a la lex artis o que no se correspondan con el supuesto de hecho previsto. ${ }^{8}$

Los dos primeros supuestos están especialmente vinculados a procesos de muerte, en concreto a la eutanasia. En cuanto al tercer límite, autores como Domínguez Luelmo (2007), recomiendan una redacción lo suficientemente elástica y dinámica de las instrucciones previas para garantizar su plena operatividad llegado el momento.

En la medida en que el plan de parto es un documento de instrucciones previas, debe ser siempre aceptado, es decir, no cabe su rechazo. Las mujeres gozan de libertad de forma en cuanto a su confección y contenido, dentro de lo establecido en la legalidad vigente.

El hecho de que el plan de parto legalmente tenga carácter jurídico de documento de instrucciones previas tiene tres consecuencias principales (Abad Villar, 2004):

a) Existe la obligación expresa de respetarlo y cumplirlo en tanto documento que contiene la autonomía y el consentimiento o la ausencia del mismo de manera formal, anticipada y por escrito.

b) Ofrece protección frente al rechazo de profesionales concretos, puesto que es el servicio al completo quien garantiza dicho respeto y cumplimiento. ${ }^{9}$ A estos efectos, Andrés Domínguez Luelmo (2007) reflexiona sobre cómo será necesario que los servicios de salud adapten y regulen sus procesos asistenciales.

c) También existe la obligación de constancia de las instrucciones previas en la historia clínica, así como de motivación expresa en caso de no cumplimiento de las mismas.

Desde una perspectiva bioética, las instrucciones previas suponen un reforzamiento del principio de autonomía de las personas usuarias y consagran el modelo moderno de relaciones sanitarias basadas íntegramente en dicha autonomía (Cantero Martínez, 2005).

\footnotetext{
${ }^{8}$ Artículo 11.3 Ley 41/2002.

${ }^{9}$ Artículo 11.2 Ley 41/2002.
} 
El plan de parto, en tanto documento de instrucciones previas, es expresión personalizada, anticipada y formal de la voluntad y autonomía de la persona. Tiene, pues, un importante contenido ético y moral, yendo más allá del consentimiento o rechazo puntual, en tanto que requiere de un proceso de toma de decisiones por parte de la usuaria (Cantero Martínez, 2005).

Dado que el documento ofrece libertad de forma y contenido, promueve la pluralidad ideológica, de conciencia y social, dando cabida a distintos modos de ver e interpretar la vida y la salud (Tarodo Soria, 2005).

Debido a las connotaciones éticas y morales, pueden darse problemas en cuanto a la interpretación de la voluntad en aquellos casos no previstos en el documento. En dichos casos, el documento puede hacer la función de guía orientadora e interpretativa (Domínguez Luelmo, 2007).

Otra de las problemáticas posibles tiene que ver precisamente con el hecho de que el respeto a la obligatoriedad de los planes de parto requiere de una gran personalización y flexibilización de la asistencia sanitaria, de difícil implantación en un contexto donde la atención y el consentimiento están estandarizados, protocolizados y burocratizados.

Respecto a los protocolos, Margarita Boladeras Cucurella (2016) reflexiona sobre cómo en teoría son útiles y deberían estar al servicio de las personas, ser fruto de la experiencia y la evidencia para ofrecer una mejor atención, pero que con frecuencia en España están obsoletos, simplifican y no responden a las necesidades de las usuarias.

El respeto a los planes de parto implica lo que Gaia Marsico (2003) llama un esfuerzo necesario por parte de las mujeres para superar la autoridad médica, para equilibrar y horizontalizar las relaciones entre profesionales y usuarias.

En este sentido, Carme Valls Llobet (2016) argumenta que solo las relaciones entre profesionales y usuarias basadas en la confianza y la amistad pueden preservar la condición de personas completas de las mujeres en lugar de convertirlas en víctimas.

Los planes de parto dan cabida a una autonomía en la que están presentes la razón y la objetividad, pero también la corporalidad, la fragilidad, la interdependencia, la vulnerabilidad o la fragilidad (Busquets Sorribas, 2016). Janet Delgado Ramírez (2016) aboga por una autonomía que no desestime la vulnerabilidad, sino que la incorpore. 
Más allá del respeto a los planes de parto, para que estos generen el impacto positivo que se espera de los mismos, son necesarias relaciones humanas de calidad, dotadas de contenido moral. Una moralidad entendida como obligación de cuidado entre las personas, donde el yo se entiende no como un ente aislado sino como un yo entre otros (Marsico, 2003).

A través del plan de parto, la atención sanitaria tiene la oportunidad de ir más allá de indicadores cuantitativos y da entrada a indicadores de mayor bienestar y calidad de vida compatibles con los mandatos salutogénicos y de promoción de la salud. En tanto que orienta la atención a personas concretas, promueve una vivencia de coherencia de las mujeres en la maternidad (Morín Fraile, 2016).

Pablo Simón (2006) considera la burocratización del consentimiento informado como una de las más grandes lacras del sistema sanitario. En la misma línea, Busquets Sorribas (2016) considera que esta burocratización implica no reconocer la importancia del consentimiento informado y conlleva continuar en un modelo basado en la beneficencia.

Herminia Biescas et al. (2017) nos alertaban de la institucionalización, estandarización y burocratización del plan de parto en contextos hospitalarios. Estas circunstancias disminuyen la capacidad de decisión de las mujeres en la medida en que no reflejan la diversidad de opciones, ni se hacen eco de necesidades concretas, centrándose únicamente en las opciones protocolizadas en el propio hospital y en ofrecer una falsa sensación de participación a las mujeres.

\section{CONCLUSIONES}

A pesar de que el plan de parto ha demostrado influir positivamente en los resultados obstétricos, hay profesionales de la salud que se sienten cuestionados profesionalmente debido a su uso, así como hospitales que institucionalizan la figura en el sentido de limitar las opciones de elección de las mujeres.

Las instrucciones previas son un instrumento legal que fomenta el ejercicio de los derechos de autonomía y consentimiento informado. Dado que el plan de parto es legalmente un documento de instrucciones previas, las mujeres tienen libertad para redactarlo en cuanto a forma y contenido, debiendo ser este aceptado desde los proveedores de la sanidad, respetándolo en la medida de lo posible y actuando la totalidad del servicio como garante de su cumplimiento. Cuando no sea posible, deberá motivarse 
en la historia clínica. En conclusión, debe reflexionarse en profundidad sobre las implicaciones jurídicas, éticas y sanitarias sobre el carácter legal de instrucciones previas del plan de parto.

\section{BIBLIOGRAFÍA}

ABAD VILLAR, Gloria. La regulación de las instrucciones previas en la ley 41/2002. En: Lizarraga Bonelli, Emilio; González Salinas, Pedro (eds.). Autonomía del paciente, información e historia clínica: (Estudios sobre la ley 41/2002 de 14 de noviembre). Madrid: Thomson-Civitas, 2004, pp. 321-364.

BIESCAS, Herminia, et al. A critical review of the birth plan use in Catalonia. En: Sexual \& Reproductive Healthcare. 2017, vol. 3, pp. 41-50.

CANTERo MARTÍNEZ, Josefa. La autonomía del paciente: del consentimiento informado al testamento vital. Albacete: Bomarzo, 2005.

CONSEJO DE EUROPA. Women's sexual and reproductive health and rights in Europe [en línea]. 2017. Disponible en:

<https://book.coe.int/eur/en/commissioner-for-human-rights/7481-pdf-womenssexual-and-reproductive-health-and-rights-in-europe.html>.

DomíngueZ Luelmo, Andrés. Derecho sanitario y responsabilidad médica. Comentarios a la ley 41/2002 e 14 de noviembre, sobre derechos del paciente, información y documentación clínica. Valladolid: Lex Nova, 2007.

FEITO, Lydia. Atención humanizada a la salud reproductiva de las mujeres. En: Boladeras Cucurella, Margarita; Goberna Tricas, Josefa (eds.). Bioética de la maternidad. Barcelona: Universidad de Barcelona, 2016, pp. 71-82.

HASENMILLER, Rose. Do Birth Plans influence Childbirth Satisfaction? [Student project]. Philadelphia: Philadelphia University, 2001.

HERNÁNDEZ RAmOs, Mario. El consentimiento informado y el derecho fundamental a la integridad física y moral. En: Sanz Mulas, Nieves (ed). Relevancia jurídica del consentimiento informado en la práctica sanitaria: responsabilidades civiles y penales. Granada: Comares, 2012, pp. 1-10.

KITZINGER, Sheila. The new good birth guide. Londres: Harmonds-worth, 1983.

LóPez EsCARCENA, Sebastian. Para escribir una tesis jurídica: Técnicas de investigación en Derecho. En: Ius et Praxis. 2011, vol. 17, núm. 1, pp. 231-246. 
LiZARRAGA Bonelli, Emilio. La información y la obtención del consentimiento en la nueva ley 41/2002 básica reguladora de la autonomía del paciente y de derechos y obligaciones en materia de información y documentación clínica. En: Lizarraga bonelli, Emilio; González Salinas, Pedro (eds.). Autonomía del paciente, información e historia clínica (Estudios sobre la ley 41/2002 de 14 de noviembre). Madrid: Thomson-Civitas, 2004, pp. 225-296.

Llamas Pombo, Eugenio. Prólogo. En: Domínguez Luelmo, Andrés. Derecho sanitario y responsabilidad médica. Comentarios a la ley 41/2002 e 14 de noviembre, sobre derechos del paciente, información y documentación clínica. Valladolid: Lex Nova, 2007.

REYNAl ReILlo, Esperanza. Consentimiento informado y responsabilidad en el ámbito sanitario. Pamplona: Ed. Aranzadi, 2017.

SANCHEZZORRILlA, Manuel. La metodología en la investigación jurídica: características peculiares y pautas generales para investigar el Derecho. En: Revista Telemática de Filosofía del Derecho. 2011, vol. 14, pp. 317-358.

SuAREZ-CORTÉS, María, et al. Uso e influencia de los planes de parto y nacimiento en el proceso de parto humanizado. En: Revista Latino-Americana de Enfermagem. 2015, vol. 23 , núm. 3, pp. 520-526. 